\title{
1 Modelling phage-bacteria interactions driving predation and horizontal gene
}

\section{2 transfer}

3

4 Jorge A. Moura de Sousa ${ }^{1,2,{ }^{*}}$, Ahlam Alsaadi ${ }^{3}$, Jakob Haaber ${ }^{3}$, Hanne Ingmer ${ }^{3}$, Eduardo P.C.

5 Rocha $^{1,2}$

$6{ }^{1}$ Microbial Evolutionary Genomics, Institut Pasteur, 28, rue Dr Roux, Paris, 75015, France.

$7 \quad{ }^{2}$ CNRS, UMR3525, 28, rue Dr Roux, Paris, 75015, France.

$8{ }^{3}$ Department of Veterinary and Animal Sciences, University of Copenhagen, Frederiksberg, Denmark

$9 *$ corresponding author: jorge-andre.sousa@pasteur.fr

10

11 SHORT TITLE: eVIVALDI: eco-evolutionary dynamics of phage-bacteria

12 Keywords: individual based modelling; predation; phage therapy; transduction; antibiotic

13 resistance. 
17 Bacteriophages shape microbial communities by predating on them and by accelerating their

18 adaptation through horizontal gene transfer. The former is the basis of phage therapy, whereas

19 the latter drives the evolution of numerous bacterial pathogens. We present a novel computational

20 approach (eVIVALDI - eco-eVolutionary mIcrobial indiViduAL-baseD sImulations) to study phage-

21 bacteria ecological interactions that integrates a large number of processes, including population

22 dynamics, environmental structure, genome evolution, and phage-mediated horizontal transfer.

23 We validate and illustrate the relevance of the model by focusing on three specific questions: the

24 ecological interactions between bacteria and virulent phage during phage and antibiotic therapy,

25 the role of prophages as competitive weapons, and how autotransduction facilitates bacterial

26 acquisition of antibiotic resistance genes upon lysis of antibiotic resistant competitors. Our model

27 recapitulates experimental and theoretical observations and provides novel insights. In particular,

28 we find that environmental structure has a strong effect on community dynamics and evolutionary

29 outcomes in all three case studies. Strong environmental structure, relative to well-mixed

30 environments and especially if antibiotics are heterogeneously distributed, enhances the rate of

31 acquisition of resistance to both phages and antibiotics, and leads to more accurate predictions of

32 the dynamics of lysogen invasion in the gastrointestinal tract. We predicted the co-existence of

33 invaders and resident lysogens in autotransduction under a range of parameters, and validated

34 this key prediction experimentally. By linking ecological and evolutionary dynamics, our modelling

35 approach sheds light on the factors that influence the dynamics of phage-bacteria interactions. It

36 can also be expanded to put forward novel hypotheses, facilitating the design of phage therapy

37 treatments and the assessment of the role of phages in the spread of antibiotic resistance. 
41 In the face of a growing threat of antibiotic resistant bacteria, bacteriophages have re-emerged as

42 a potential alternative to clinical treatments of infections, as they are efficient bacterial predators.

43 However, bacteriophages can also promote, through a mechanism called transduction, the

44 dissemination of adaptive traits between bacteria, including antibiotic resistance genes.

45 Importantly, these two types of interactions (predation and transduction) can co-occur, which

46 creates difficulties in predicting their outcome. We have developed eVIVALDI (eco-eVolutionary

47 mlcrobial indiViduAL-baseD slmulations), a computational model that allows the simulation of

48 microbial communities with a focus on the mechanisms involved in phage-bacteria interactions,

49 across time and in different types of environments. eVIVALDI can be used to understand the

50 conditions where phages are more likely to be successfully used to eliminate bacteria or, in the

51 other hand, the conditions where they increase the probability of dissemination of adaptive traits.

52 Our research highlights the importance of considering the diverse ways that phage and bacteria

53 interact, and the relevant ecological conditions where these interactions take place, to understand

54 how bacteriophages shape microbial communities and how they can be used as a clinical tool. 


\section{Introduction}

56 Microbial organisms are pervasive across all natural environments, including the human body.

57 Their adaptation and organization in communities may lead to disease [1], drive host evolution [2],

58 and produce major changes in ecosystems [3,4]. Ecological interactions in microbial communities

59 influence, and are influenced by, the rapid pace with which microbes acquire adaptive changes

$60[5,6]$. A striking example is the relationship between bacteria and bacteriophages (from here on

61 referred to as phages), because the latter predate on the former whilst also driving their adaptation

62 [4]. Phages are the most abundant entities in nature $[7,8]$ and very efficient bacterial predators; it

63 has been estimated that they promote the turnover of $\sim 20 \%$ of bacterial mass every single day in

64 certain environments $[9,10]$. In the context of widespread antibiotic resistance, this has led to a

65 rekindled interest in phage therapy as an adjuvant or a replacement of antibiotic therapy against

66 multi-resistant bacteria [11].

67 Virulent phages follow a strictly lytic cycle within their hosts, but they often exist in diverse

68 communities with other virulent and temperate phages. Infection by the latter can lead to either

69 the lytic cycle or their integration in bacterial genomes, as prophages (lysogenic cycle). Temperate

70 phages are not used for phage therapy because lysogeny prevents them from extinguishing

71 bacterial populations and confers resistance to closely, and sometimes distantly related phages -

72 a mechanisms known as superinfection exclusion [12-14]. However, half of all bacterial genomes

73 contain at least one, and up to 20 , prophages, with these being more frequently found in bacterial

74 pathogens [15], which means that they cannot be ignored in phage therapy. The expression of

75 prophage genes may provide novel traits to the host (lysogenic conversion), and many cases have

76 been described where prophages carry adaptive traits implicated in virulence or resistance to

77 stress [16]. Virions arising from prophage induction can infect closely related competitor bacteria

78 that are non-lysogenic for the phage, decreasing bacterial competition, increasing prophage 
79 frequency, and liberating resources that can be used for the growth of the remaining lysogenic

80 population [17]. In this case, prophages have been regarded as weapons against bacterial

81 competitors $[13,18]$.

82 Phages can drive horizontal gene transfer between bacteria by transduction [19]. This can be a

83 hazard in the case of phage therapy if the transferred traits are virulence factors or antibiotic

84 resistance genes. Specialized transduction occurs in temperate phages when prophage excision

85 leads to the transfer of neighboring chromosomal genes. Generalized transduction occurs when

86 bacterial DNA is delivered to other cells after being encapsulated in virions, due to the specificities

87 of the pac DNA packaging system [20]. Although these mechanisms are commonly used as

88 genetic engineering tools [21], they have been considered inevitable consequences of errors in

89 phage replication machineries and their rates in nature are poorly known. In the lab, they vary

90 across several orders of magnitude (between $10^{-11}$ and $10^{-3}[22,23]$ ), depending on the phage, the

91 environment, and the type of culture media [19]. Importantly, phage driven transmission of

92 bacterial DNA can have particularly nefarious consequences for humans. Transducing phages are

93 responsible for the transmission of virulence factors in Staphylococcus aureus [24], and may

94 accelerate the spread of antibiotic resistance genes $[25,26]$. Transduction can also have an impact

95 at very short time scales: prophage induction facilitates the capture of adaptive traits (e.g., an

96 antibiotic resistance gene) from a second bacterial strain that is infected by the phage and, through

97 generalized transduction, transfers the gene back to the lysogenic strain. This process has been

98 called autotransduction [27]. Hence, phages drive the evolution of bacterial gene repertoires and

99 may spread virulence or antibiotic resistance factors during phage therapy.

100 The diversity of interactions between phages and bacteria may obscure the effects of each of 101 them. Experimental approaches have described and clarified the mechanisms underlying these 102 interactions, but usually focused on simplified environments [8,28]. In vivo studies of these 103 interactions (e.g., in mammalian hosts [29]) tackle more natural environments, but have limited 
resolution in tracking temporal dynamics or the effects of individual mechanisms. Mathematical modelling provides a complementary approach to the study of phage-bacteria interactions, providing important insights on their co-evolutionary processes [30] or the dynamics of particular bacterial defense mechanisms [31,32]. Previous models have focused on individual mechanisms

108 of interaction in simple environments (e.g., how the evolution of resistance to phage can affect 109 clinical treatments [33]), because tackling multiple mechanisms and spatial heterogeneity hinders

110 the development of analytical solutions. Yet, natural communities, and particularly those relevant

111 for phage therapy, include complex interactions and spatial structure [34-38]. This may explain

112 why models sometimes fail to fully reproduce in vivo dynamics of phage infection [28], and why

113 there is paucity of models on the impact of phage-mediated horizontal gene transfer in the

114 adaptation of bacterial communities (but see the work of Volkova et al. [39] for a theoretical

115 comparison between the relative efficacy of transduction versus conjugation in transmitting an

116 adaptive trait).

117 Individual-based models (IBMs) are an alternative to population-based mathematical approaches

118 for studying complex microbial systems [40]. They have been useful to understand, for instance,

119 the effect of spatial structure in microbial social evolution [41] or the interactions between bacteria

120 and virulent phages in biofilms [36]. Although computationally intensive, IBMs provide a framework

121 to study biological systems through the incorporation of different (and potentially interacting)

122 mechanisms at the level of the individual. Population-level dynamics can then emerge from the

123 collective individual behaviors. This makes IBMs particularly appealing to investigate phage-

124 bacteria interactions, because these involve both ecological (e.g., predation) and evolutionary

125 (e.g., transduction of adaptive traits) scales, with antagonistic mechanisms defined at the 126 individual level (e.g., the lysis-lysogeny decision of temperate phage). To study these multiple 127 roles of phages in microbial communities, we developed an IBM approach that is able to simulate 128 diverse mechanisms and eco-evolutionary contexts: eVIVALDI - eco-eVolutionary mlcrobial 
129 indiViduAL-baseD slmulations. We focus on three questions, of gradually increasing complexity,

130 that are relevant for bacterial evolution and phage therapy, and that cover a range of possible eco-

131 evolutionary interactions between bacteria and phage. First, we introduce the basic scheme of the

132 simulation with the study of ecological interactions between co-evolving virulent phages and

133 bacteria under phage and antibiotic pressure in structured environments. Then we introduce

134 lysogeny and super-infection exclusion in the model to study the role of prophages as competitive

135 weapons. We show that our model provides better fit to previous experimental results than earlier

136 models. Finally, we introduce transduction and the way we encode individual genomes in the

137 model to elucidate how bacteria may obtain novel adaptive genes from sensitive bacteria by

138 autotransduction. We use eVIVALDI to explore and quantify the different mechanisms of phage-

139 bacteria interactions and to gain insights on how the structure of the environment can affect these

140 interactions and the community dynamics. We tackle each question by demonstrating the ability

141 of the model to capture previous results and then show how its complexity highlights new relevant

142 features. 


\section{Methods}

\section{Concept and basic implementation}

145 The eVIVALDI model was developed in Python (version 2.7.3), using an object-oriented approach, 146 with a focus on the flexibility and extensibility of mechanisms and parameters simulated. The 147 complete ODD (Overview, Design concepts, and Details) protocol [42] of the developed model is 148 available as supplementary text (Text S1, Table S1 has the parameters modelled and their 149 possible values), but below is a brief overview of the model. The source of the software can be 150 obtained in the following link: https://gitlab. pasteur.fr/jsousa/eVIVALDI. The simulations can be run 151 on a typical desktop computer. In a 3GHz 8-core Mac Pro, with 32GB of RAM, a replicate of a 152 simulation (100 iterations), takes from $\sim 5$ to 30 minutes, depending on the parameters. 153 Computations can also be performed in a cluster, allowing the parallel simulation of multiple 154 parameters.

\section{Entities and their ecological setup}

156 Both bacterial cells and phage particles are represented as independent individuals on an 157 environment represented as a two-dimensional grid with Moore neighbourhood (the 8 connected 158 grid spaces of each location, for a Moore distance of one) (Fig 1A). Bacteria can be of different 159 species. Each individual bacterium has a genome with core, accessory and, eventually, prophage 160 genes. Bacteria have individual phenotypes, such as growth rate or the ability to survive antibiotic 161 exposure. Phages can be from different species, have different lifestyles (temperate, virulent or 162 defective), and possess individual phenotypes (e.g., attachment receptors and burst sizes). The 163 host range of phage hosts is defined by a matrix (Fig 1B), and the superinfection exclusion rules 164 amongst phages is defined in a similar way (Fig 1C). 


\section{Environmental and bacterial updates}

166 The environment and the individuals are updated and behave according to biologically inspired

167 rules. The environment can be completely structured, semi-structured or not structured at all (i.e., 168 well-mixed), and it can be set as bounded or have a toroidal space. The type of structure influences 169 the diffusion of the different bacterial cells and environmental particles (phage and antibiotics). 170 Each location can hold a single bacterial cell and several phage cells. Free space is the bacterial 171 resource to be consumed, and it is freed whenever bacteria die. Bacterial death can be 172 intrinsic (e.g., of old age) or explicit (e.g., exposure to antibiotics or predation by phage) (Fig 1D). 173 When a free space is available, the neighboring bacteria compete for reproduction. The outcome 174 of the competition is chosen through a roulette wheel method that accounts for the fitness of each 175 bacterium. The successful bacterium generates an offspring into the free space (Fig 1E). Bacteria 176 can be infected by phage in the environment. The outcome of the infection depends on the phage 177 lifestyle and, for temperate phage, the lysis-lysogeny decision. This decision is stochastic but 178 influenced by the number of surrounding phages. For temperate phage, integration in the host 179 genome means vertical inheritance with host replication, until the phage excises from the genome, 180 according to a probability that can be low but non-null throughout the simulation (stochastic 181 prophage induction) and that can also be influenced by the level of antibiotic stress to which the 182 host is exposed. Phage can transduce bacterial genes to other bacteria by generalized or 183 specialized transduction (depending on phages' characteristics, Fig 1F).

185 The inputs of each simulation are two text files that define the general parameters and also the 186 ecological setup of the environment (types and numbers of bacteria and/or phage, along with their 187 attributes). The statistics collected at different time points are stored in dictionaries and dataframes 188 (using pandas), can be tailored to the experimenter's choice and can be represented visually 
189 (using matplotlib and seaborn) or created as an output file.

\section{Random Forest Analysis}

191 The Random Forest Analysis is based on simulations performed with the model, covering 3000

192 random combinations of parameters, with 30 simulated repeats per combination. The output of

193 this cohort of simulations is grouped and resumed in response variables, to which a column with

1943000 rows of a random parameter is added (i.e., a choice of a number between 1 and 3 ). This

195 table is used as input of the randomForest package in $\mathrm{R}$ (version 4.6.12), where the randomForest

196 function is run with the parameters ntrees set to 10000. The relative importance of each parameter

197 (the percentage increase in minimum squared error, \%IncMSE) is assessed using the importance

198 function from the same package.

199 Bacterial strains, antibiotics and growth conditions

200 Bacteria strains used in this study are listed in Table S2. Bacteria were grown in Tryptic Soy Broth

201 (TSB), Tryptic Soy Agar (TSA) and TSB+0,04\%TSA from Oxiod. When appropriate, the following

202 antibiotics were applied: Erythromycin 10mg/L, Chloramphenicol $10 \mathrm{mg} / \mathrm{L}$, Streptomycin $50 \mathrm{mg} / \mathrm{L}$,

$2030.5 \mathrm{mg} / \mathrm{L}$ of Rifampicin. All antibiotics were purchased from Sigma.

204 Co-culture experiment

205 Cultures of JH944, JH927 and JH930 were incubated in 20ml TSB overnight with shaking (200

$206 \mathrm{rpm}$ ) at $37 \cdot \mathrm{C}$. OD600 was measured and cultures were diluted to final OD600 $=0.01$ in $1: 1$ ratios

207 of $\mathrm{JH} 944+\mathrm{JH} 930$ and $\mathrm{JH} 944+\mathrm{JH} 927$ in TSB media supplemented with $5 \mathrm{mM} \mathrm{CaCl}$. After overnight

208 incubation (shaking $200 \mathrm{rpm}$ at $37 \circ \mathrm{C}$ ), the cultures were sonicated (10 pulses, 500msec, 50\%) to

209 ensure single bacterial cells when plating. Serially diluted cultures were plated on TSA containing

210 erythromycin (

$21137 \circ \mathrm{C}$. The following day, 100 single colonies from the $\mathrm{JH} 944+\mathrm{JH} 927$ culture plates were streaked 
212 on TSA supplemented with streptomycin and rifampicin, TSA supplemented with erythromycin 213 and TSA without addition. For the JH944+JH930 culture, 100 colonies were restreaked on TSA 214 supplemented with streptomycin and rifampicin, TSA supplemented with chloramphenicol and 215 TSA without addition. All plates were incubated overnight at $37 \circ \mathrm{C}$.

\section{Phage induction assay}

217 Bacteria lysogenic for phi11 were detected by a prophage induction assay. Bacterial colonies that were present on TSA supplemented with chloramphenicol or erythromycin but absent on TSA supplemented with streptomycin and rifampicin were selected for the phage induction assay. The bacteria were inoculated in TSB and grown to mid-log phase $\left(\mathrm{OD}_{600}=0.5\right)$ with shaking $(200 \mathrm{rpm})$ at $37 \circ \mathrm{C}$. Mitomycin C (Sigma) $0,4 \mathrm{mg} / \mathrm{L}$ was added to TSB to induce any prophages present in the 222 strain and the cultures were incubated at $37 \circ \mathrm{C}$ with shaking ( $200 \mathrm{rpm})$ overnight. The cultures 223 were centrifuged at $4 \circ \mathrm{C}, 3700 \mathrm{rpm}$ for $10 \mathrm{~min}$ to pellet the cells and supernatant sterile filtered $224(0.22 \mu \mathrm{m})$. Phages present in the supernatant were detected by mixing $100 \mu \mathrm{l}$ of the filtered 225 supernatant and $100 \mu \mathrm{l}$ of the indicator strain RN4220 in presence of $15 \mu \mathrm{l} 100 \mathrm{mM} \mathrm{CalCl} 2 . \mathrm{After}$ 226 incubation at room temperature for $10 \mathrm{~min}$, top agar (TSB+0,04\% TSA) was added and the mixture 227 was poured onto a TSA plate supplemented with $10 \mu \mathrm{M} \mathrm{CaCl}_{2}$ and incubated at $37 \circ \mathrm{C}$ overnight. 228 Phages present in the plates were indicative of the original culture being lysogenized by the phage. phages. The environment ranges from completely well-mixed (liquid), where the contents of each location are randomized at each

233 iteration, to spatially structured, where they are fixed. An intermediate structure is achieved by allowing replication of bacterial cells

234 into a neighbourhood of a given distance. Bacteria and phage can be of different species, and the latter exist as entities either in the 
bioRxiv preprint doi: https://doi org/10.1101/291328; this version posted April 16, 2018. The copyright holder for this preprint (which was not

certified by peer review) is the author/funder, who has granted bioRxiv a license to display the preprint in perpetuity. It is made available under aCC-BY-ND 4.0 International license.

death can increase by antibiotic exposure or phage infection. Phages decay in function of the period of time spent outside a bacterial host. E) Bacteria compete to reproduce to empty locations, with the fittest bacteria being more likely to produce an offspring. The

240 offspring inherits the traits of the parent cell, but can undergo mutations and is placed into the free location. F) The type of phage

241 infection is determined by the lifestyle of the phage, with virulent following an obligatory lytic cycle, whilst temperate phage can

242 undertake the lytic or the lysogenic cycle following a stochastic decision affected by the density of phages in the environment. The

243 probability of specialized transduction is computed during excision, leading eventually to the incorporation into the phage DNA of a

244 neighboring gene. Generalized transduction occurs before the burst, and a virion has the probability to incorporate random genes from

245 its host, instead of its own DNA. Transduced genes can be used by the subsequently infected bacterial hosts. G) The main cycle of a

246 typical simulation within the model. See complete ODD in Supplementary Material. 


\section{Ecology of phage-bacteria interactions in the light of antibiotic and phage therapy}

249 Antimicrobial therapies rely on the effectiveness of selective agents to kill sensitive bacteria.

250 Phage therapy involves infection and reproduction of the killing agents, thus extending the ability

251 of standard chemical therapies. We started by investigating if eVIVALDI could reproduce simplified

252 but typical ecological scenarios where sensitive individuals are killed by antibiotics and/or predated

253 by virulent phages, thus promoting the increase in frequency of resistant bacteria. A simple

254 community of two bacterial species, one sensitive and another resistant (either to antibiotic or

255 virulent phage), was simulated in a well-mixed environment, and no new resistant bacterial

256 mutants were allowed to emerge in these simulations. Resistance can be defined as costly, in line

257 with experimental data [43], rendering resistant bacteria less competitive in the absence of

258 selection pressure (Fig S1). However, when either antibiotics (Fig 2A) or phage (Fig 2B) were

259 introduced in the environment, the resistant population rapidly increases to fixation. Predation by

260 phage leads to an initial increase in their numbers, because of the abundance of sensitive bacteria,

261 but also to their subsequent rapid extinction when sensitive hosts become unavailable (Fig 2B). A

262 combined treatment of antibiotics and virulent phages leads to the extinction of both populations

263 because none has the ability to survive both selective pressures (Fig 2C). However, the decrease

264 of the antibiotic sensitive population is slower in the presence of phages because of lower

265 competition from antibiotic resistant cells, which are killed by the phage (Fig S2A).

266 Our model allows to test explicitly the effect of spatial structure on community composition. Spatial

267 structure affects the ability of individuals to diffuse freely in the environment and is known to affect

268 population dynamics [35,36]. Here, spatially structured environments assume that bacteria are

269 fixed in their locations, and can divide only to their immediately adjacent locations. Likewise, we

270 assume that, in these environments, phage cannot diffuse, and thus can only propagate by 
271 infecting nearby bacteria. Antibiotics applied homogeneously in spatially structured communities

272 delay the extinction of the sensitive bacteria in comparison to non-structured environments (Fig

273 S2B). However, antibiotics are more likely to be applied non-homogeneously when environments

274 are structured. The delayed extinction is more pronounced in these conditions leading to long term

275 coexistence between sensitive and resistance bacteria (Fig S3). The effect of phage predation on

276 community dynamics is markedly different between well-mixed and spatially structured

277 environments because the latter decreases dispersion leading to "predation waves" that produce

278 spatial arrangements of dead cells akin to those observed in phage plaque assays (see Fig S4

279 and Video S1). Ultimately, spatial structure results in delayed extinction of phage susceptible cells

280 (Fig 2E vs Fig 2B). Similar to well-mixed environments, presence of antibiotics and phage in

281 spatially structured environments leads to a much slower extinction of antibiotic resistant bacteria

282 compared to environments with antibiotics but lacking phages (Fig 2F vs Fig 2D). However, the

283 presence of phages and antibiotics in spatially structured environments leads to a faster extinction

284 of antibiotic sensitive populations, compared to well mixed environments (Fig 2F vs Fig 2C, Fig

285 S2C), due to a much less efficient phage predation of their competitors when the environment is

286 structured.

Fig 2. Community dynamics driven by antibiotic selection and phage predation. A small community composed of two different species is subjected to different selective pressures. Bacteria can be sensitive to antibiotics but resistant to phage (in green), or resistant to antibiotics but sensitive to phage (in red). We follow the temporal dynamics and show the populations in their respective colors (the number of free phage in the environment is shown in black). Solid lines indicate mean values for 30 simulations ran with

292 the same parameters and shaded areas show their 95\% confidence interval. At the right of each plot is a representative time lapse at

2933 time points of the lattices for each scenario, where the colors represent each bacterial species and white spaces represent the

294 absence of bacterial cells. In A) and D) antibiotics are applied at the indicated time. In B) and E), virulent phages (10 individual particles)

295 are co-inoculated with the bacteria at time $0 . \ln$ C) and F), both selective regimes are applied, with antibiotics applied at the indicated 296 time and virulent phage co-inoculated with bacteria at time 0. In A), B) and C), the environment is homogeneous (well-mixed), as in 297 liquid culture. In D), E) and F), the environment is spatially structured. In D) and F) antibiotics are applied homogenously in the 
structured environment, and in E) and F) each of the 10 phage particles is initially placed randomly in the biofilm. The complete set of parameters for these simulations is show in supplementary data.

301 The introduction of mutations in the model, eventually reversing the susceptibility to antibiotics or 302 phages, tends to stabilize the bacterial populations (Fig S5A-B). Nevertheless, some populations 303 still go extinct because of the loss of rare mutants by genetic drift or because no adaptive 304 mutations occurred in the time span. Under pressure of antibiotics and phages, double resistant 305 cells emerge only when the mutation rate is very high (Fig S5C). The impact of the environmental 306 structure in the dynamics of predation (Fig 2) led us to analyze how it affects the emergence of 307 resistant lineages (Fig 3). Whilst single resistant mutants increase in frequency slower in 308 structured environments (Fig S5D), double mutants resistant to antibiotics and phage are much 309 more likely to emerge (Fig 3A-D) for intermediate rates of mutation (Fig 3E). This is because in 310 structured environments, the rare mutants resistant to antibiotics benefit from the resources 311 available from neighboring dead cells and rise in frequency without contact with phages (that 312 diffuse less efficiently). This increases the span of time available for the acquisition of secondary 313 mutations conferring resistance to phages, especially if the initial number of phages is not very 314 high (Fig S6). Hence, the acquisition of multiple adaptive mutations is more likely to occur in 315 structured environments. sensitive to antibiotics and phage. Lines show 30 replicate simulations with emerging resistant lineages (to one or both selective pressures). Single mutants resistant to phage are shown in red, whilst single mutants resistant to bacteria are shown in green. Double mutant lineages resistant to antibiotics and phage are shown in grey. In A) and B) mutants emerge at a rate of $10^{-4} . C$ and D) mutants

321 emerge at a rate of $10^{-3}$. A and $C$ show dynamics from well-mixed environments. $B$ and $D$ show dynamics from spatially structured

322 environments. E) Percentage of simulations (out of 30) where lineages resistant both to antibiotics and phage have emerged, in either 323 well mixed or spatially structured environments, for all the mutation rates tested (x-axis). The complete set of parameters for these 
simulations is show in supplementary data.

326 The ability of bacteria to evolve resistance to phage might be futile if phage can also adapt 327 sufficiently fast to overcome these changes [44]. When we allowed bacteria and phage to evolve 328 in our simulations (Fig S7A), we observed co-evolutionary arms races similar to both theoretical 329 expectations [33] and experimental observations [45]. Spatially structured environments showed 330 slower co-evolution dynamics and higher variability between simulations than well-mixed ones 331 (Fig S7B). Heterogeneous antibiotics added in structured environments further delayed the co332 evolution dynamics (Fig S7C-D), due to the death of a significant part of the bacterial population.

333 Importantly, and as before (Fig 3), surviving bacteria (either resistant to antibiotics or not exposed

334 to lethal concentrations) were able to generate mutants resistant to phages for a longer period of 335 time. This is not only due to the limited diffusion of phage, but also because phages need bacterial 336 hosts to replicate and to generate their own genetic diversity. Thus, a reduction in the number of 337 bacterial hosts due to antibiotic exposure hinders both phage propagation and evolution. This 338 suggests that, in natural environments, multiple stressors might render co-evolutionary arms races 339 less predictable than proposed by theoretical models and experimental settings that assume 340 homogeneous populations and environments.

341 Lysogeny as a weapon

342 Contrary to virulent phages, temperate phages may integrate the bacterial host genome and 343 reproduce vertically with it. The lysis-lysogeny decision in our simulations mimics experimental 344 observations [46], and is influenced by the amount of competition faced by the phage: lysogeny is 345 more likely under high viral concentrations or high multiplicity of infection (Fig S8). Since lysogens 346 are protected from further infections by similar phages, due to superinfection exclusion, the 347 environmental concentration of phages in the simulations decreases rapidly with the increase of 
348 lysogens (and depending on free phage half-life). When lysogeny occurs mostly at high viral 349 concentration the bacterial population can become extinct before lysogens can arise. 350 Theoretically, this can also result in the extinction of the phage population.

351 When a lysogen invader arrives at a community with resident bacteria sensitive to its prophage, 352 Iysis of a small fraction of the invaders can dramatically reduce the population of resident sensitive 353 bacteria. This liberates resources for the lysogenic invaders [13]. eVIVALDI recapitulates previous 354 experimental data on this prophage-as-a-weapon hypothesis [17] (Fig 4a): prophage induction 355 rapidly decreases the sensitive population of residents in the early stages of the process, but 356 lysogenization of the latter rapidly neutralizes this process (because the resident lysogens are 357 now resistant to the phage). Hence, the use of prophages as a biological weapon can provide a 358 decisive advantage for colonizing a new niche, but is rapidly neutralized by lysogenization of 359 competitor bacteria. This is also in agreement with previous theoretical works exploring dynamics 360 of invasion in well-mixed environments, using prophages as a competitive weapon [47].

361 The advantage of lysogens in the colonization of an environment of resident sensitive bacteria 362 was recently demonstrated in the mouse gut and was suggested to depend on the initial ratio 363 between invaders and resident cells [29]. Indeed, our simulations considering different initial ratios 364 of invading lysogens versus resident non-lysogens showed that the latter were more likely to 365 survive as lysogens when more abundant in the beginning of the process (Fig S9). The 366 abovementioned study presented a population-based mathematical model that fitted well most 367 experimental data, but predicted faster initial infection rates than the observed ones. While 368 different parameters can slow down these dynamics (e.g., the burst size of the phage [29]), the 369 spatially structured mouse gastrointestinal tract is likely to interfere with the temporal dynamics of 370 lysogeny. Interestingly, the inclusion of spatial structure in our model, absent from the 371 abovementioned models, led to a slower increase of free viral particles and slower generation of 372 lysogens in the resident strain (Fig 4B-C). This implicates that invading lysogens may be more 
Fig 4. The role of lysogeny in community dynamics. A) Genomes from species A (invaders, grey bars) carry an inducible prophage,

whereas those of species B (residents, white bars) are initially non-lysogens. Species are co-inoculated at a 1:10 mixture. Phages (black lines) are spontaneously induced from the lysogenic population. These phages infect the sensitive resident population, which may form lysogens that are protected from phages ( $\mathrm{B}^{*}$, black bars). Eventually, the resident that are not lysogens become extinct. All bars represent the average of 30 replicate simulations with similar parameters, with the error bars indicating their $95 \%$ confidence interval. Data was displayed as in Figure 1 of [17] for comparison. B) Invading lysogens (L, red lines) and resident sensitive cells (S, blue lines) are co-inoculated at a ratio of 1:10. Phages (purple lines) are spontaneously induced and generate new lysogens in the sensitive resident cells $\left(\mathrm{S}^{\mathrm{L}}\right.$, green lines). Full lines: well-mixed environments. Dashed lines: spatially structured environment. Data was displayed as in Figure 3 of [29] for comparison. C) Emergence of resident lysogens in well-mixed (blue) and in spatially structured (orange) environments during the initial 10 iterations of the simulations shown in B. Shown is the polynomial fit of order 2 for the initial 10 iterations, for each of the two types of environment; ANCOVA between the two environments, $F=485.5, p=0$. The complete set of parameters for these simulations is show in supplementary data.

394 population sizes: a non-lysogenic strain resistant to antibiotics ("residents") and a strain of 395 lysogenic antibiotic sensitive "invaders" (Fig 5A). After initial growth, antibiotics are applied in the 396 environment and, as in the experimental study [27], the invaders survive because they acquire the 397 resistance gene by generalized transduction (Fig 5A-B). The analysis of the bacterial genomes in 398 the simulations indicates multiple successive transduction events from the resident to the invader 399 cells (Fig 5C). These events are random (i.e., transduction can transfer any part of bacterial DNA), 
but natural selection results in over-representation of those transferring antibiotic resistance

401 genes. Overall, invaders lead residents to extinction in most simulations (62\%), but sometimes residents become lysogens and outcompete invaders (3\%). Interestingly, many simulations exhibited coexistence of lysogenic invaders and lysogenic residents (22\%, Fig 5D), and a few showed extinction of all bacterial populations (13\%).

Fig 5. Simulation of autotransduction. A) Representation of the autotransduction events. We created a multispecies community akin to the experimental work of [27], where the invader lysogenic species (red) is sensitive to antibiotics and the resident non-lysogenic species (green) is resistant to antibiotics but sensitive to the phage of the invaders. B) Temporal dynamics of a typical simulation leading to the survival of the invaders. The black line indicates the number of phages in the environment and the time of application of antibiotics is indicated with the grey line. C) Samples of genomes in the population at two different time points of the simulations of panel B. Before antibiotics $(t=2)$, the genomes of the resident population (green) carry the resistance trait (orange marker). The invader population (red) is not resistant (grey marker indicates sensitivity to drugs). After the application of antibiotic $(t=10)$ most of the invaders have the original prophage and a random sequence of bacterial DNA transduced from the resident cells (other ellipses). D) Outcome of 100 simulations. The complete set of parameters for these simulations is show in supplementary data.

eVIVALDI includes many complex stochastic mechanisms and it is not straightforward to

417 empirically disentangle the importance of each in the final outcome. Therefore, we used a machine 418 learning approach, Random Forest Analysis (RFA, see Methods), to quantify the importance of 419 the mechanisms driving the increase of the population of invaders (Fig 6A, in File S7 are the 420 parameters explored with the RFA). We focused on the percentage increase in minimum squared 421 error (MSE) associated with each variable in the simulation. Generalized transduction had the 422 strongest effect in the efficiency of autotransduction (86\% increase in mean square error [MSE], 423 Fig 6B), whilst specialized transduction was almost negligible ( $3 \%$ increase in MSE). 424 Autotransduction also improved with higher probability of adsorption (44\% increase in MSE) and 425 infection distance (i.e., the maximum distance between a bacterium and a phage still allowing 
426 infection, $70 \%$ increase in MSE), because they increase the reach and efficiency of infection by

427 phage and, subsequently, the likelihood of generalized transduction (Fig 6C, Fig S11B). In

428 contrast, when the decision to enter lysogeny ( $49 \%$ increase in MSE) can be made with high

429 probability for relatively low viral concentrations, the resident population proliferates (Fig S11D

430 and Fig S11I for the lysis-lysogeny decision functions explored with the RFA). The importance of

431 the remaining parameters is detailed in Fig S11.

Fig 6. Identification of the main mechanisms affecting the rate of autotransduction of an antibiotic resistance gene using

Random Forest Analysis. A) Analysis is based on 3000 randomized combinations of parameters and 30 repeated simulations for each combination). Parameters with a higher $\%$ in increased minimum square error have a higher importance for the measured outcome: the median of the final relative frequency of the invader. A random parameter (in grey) was included in the analysis to provide a baseline reference of importance. B-C) The directionality of the impact of two parameters is assessed by plotting the frequency of the invader population at the end of the simulation (across all simulations), in function of the parameter of interest (the other parameters are shown in Fig S11). In the left y-axis, and as strip plot of grey dots, is the distribution of the frequency of the invader population in all simulations. In the right $y$-axis, and as red dots and lines, is the median of this frequency across the simulations.

442 To better understand the relationship between two of the most important parameters, generalized

443 transduction and probability of phage attachment, we explored their space of parameters at a

444 higher resolution than before (Fig 7A), while fixing all other parameters. We found that a critical

445 combination of high adsorption efficiency (>1\%) and high (between $0.01 \%$ and $80 \%$ ) probability

446 of generalized transduction is required for the survival of invaders (red region). The survival of the

447 resident population is the most likely outcome when rates of transduction and/or infection are low,

448 but also when all phages engage in generalized transduction (100\% probability of generalized

449 transduction), because in this case no viable particles are released in the environment (green

450 region). The space of parameters leading to coexistence (yellow region) separates the region

451 leading to the overrepresentation of the invaders from the one leading to the overrepresentation 
of the resident species (see also Fig S12).

453 The study describing the discovery of autotransduction focused on the process of gene acquisition

454 by the invaders and did not address the possibility of co-existence [27]. We thus experimentally

455 addressed the prediction of co-existence by co-culturing in liquid media supplemented with

456 chloramphenicol two strains of Staphylococcus aureus: a lysogenic strain (JH944, "invaders")

457 sensitive to the antibiotic and either one of two non-lysogenic strains (JH930 or JH927, "residents")

458 resistant to the antibiotic (see Methods). The two different resident strains were chosen to

459 demonstrate the result both in the laboratory strain 8325-4 (JH930) as well as in the clinically more

460 relevant strain USA300 (JH927) background. The majority of the colonies resistant to

461 chloramphenicol isolated at the end of the two experiments are in the JH944 background,

462 indicating the acquisition of the resistance gene from the resident bacteria by autotransduction.

463 However, in both combination of strains, performed in 3 biological replicates, a subpopulation of

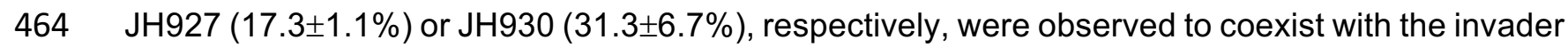

465 strain (Fig 7B, Table S3), confirming our predictions. Interestingly, in simulations were coexistence

466 is frequently observed, the frequency of invaders can be influenced by both the probability of

467 adsorption and the probability of generalized transduction (Fig S13). The different frequencies of

468 invaders observed in co-culture with either JH927 or JH930 could represent different regions of

469 the predicted parameter space, where released phages have, for instance, a higher infection

470 efficiency towards JH927 compared to JH930. Confirmation of this hypothesis will require further

471 work on the biology of these phages. Importantly, we observed in the simulations that coexistence

472 is strictly dependent on the generation of lysogenic variants of the resident bacteria, being

473 suppressed when we performed simulations without the generation of new lysogens (Fig S14A-

474 D). This is experimentally corroborated with the observed release of phage particles from the

475 surviving resident clones at the end of the co-culture, when these are exposed to mitomycin C

476 (Fig 7B, Table S3, see Methods). This confirms that lysogenization of the resident bacteria is the 
477 mechanism responsible for the coexistence between the two strains and validates the predictions

478 of the model.

479 Our simulations further suggest that structured environments (Fig S12A) provide an additional 480 region, for extremely high rates of transduction, where coexistence is prevalent (upper regions in 481 Fig S12B-C). These high rates are biologically implausible for viable phages, but not for defective 482 phages or for gene transfer agents [48]. Finally, extinctions of both strains were more frequent 483 when the probability of adsorption was high and transduction was low, suggesting that an inducible 484 phage that is highly infective but a poor transducer is more likely to lead to the collapse of both 485 the invaders and the antibiotic resistant populations. The likelihood of double extinctions is higher 486 in structured environments (Fig S12A, Fig S15 and Fig S16) or in the absence of lysogenization 487 of the resident bacteria (Fig S14E-F and Fig S17). Our results suggest that ecological interactions 488 between strains invading communities of susceptible bacteria can be very diverse, depending on 489 the rates of infection, transduction, lysogenization and population structure. resistance gene. The simulation scenario is similar to Fig 6. A) The heatmap represents the likelihood of the outcome of the simulations in function of the two parameters. The color scale ranges from green $(100 \%$ of the final population composed resident bacteria) to red ( $100 \%$ of the final population composed of invader bacteria), with yellow regions indicating cases where coexistence is the outcome more likely to occur in the timeframe of the simulations. 30 repeat simulations were performed for each combination of parameters, and their median value is used to construct the heatmap. When both populations went extinct, this was either ignored to compute the median (if it occurred in less than $50 \%$ of the cases), or was marked as grey (otherwise). B) Co-cultures of a lysogenic chloramphenicol sensitive strain of S. aureus (JH944, "invaders", red) and a non-lysogenic chloramphenicol resistant strain (JH930 or JH927, "residents", green or green with white circles, respectively) indicate coexistence between the two strains at the end of the 
504 Individual-based modelling is providing novel ways to analyze and predict the behavior of microbial

505 systems [40]. Our novel approach integrates multiple and different bacterial species, phages,

506 environmental structures and ecological conditions to explore different aspects of bacteria-phage

507 interactions: temporal changes in community composition (e.g., between lysogens and non-

508 lysogenic bacteria), the concurrent effects of mechanisms of infection, lysogeny, and transduction,

509 and their consequences for the genomic composition of each individual bacteria and phage. To

510 the best of our knowledge, no other theoretical or computation model integrates these different

511 scales of phage-bacteria interactions. This has allowed us to characterize and quantify key

512 ecological components, such as structured environments, in the dynamics emerging from these

513 interactions.

514 Models are based on simplifying assumptions to make biological systems more tractable. This

515 facilitates pinpointing the relevance of certain mechanisms or agents, but may result in misleading

516 over-simplifications of the system. One major difference between biological systems and our

517 model concerns the number of cells which, due to computational reasons, is lower than the one

518 typically used in experimental settings. Even though our results are qualitatively similar to

519 experimental and/or other theoretical works, this difference may affect the quantitative results. The

520 decreased effective population size (and the consequent increase in the effect of drift) requires

521 that certain rates (e.g., mutation or transduction rates) are simulated at higher values, in order to

522 increase the probability of detecting such events. Another limitation lies in the characterization of

523 the environment. Even if we allow for different levels of structure, environments are spatially and

524 temporally constant throughout the simulations, which might not always be the case in nature.

525 This can change the dynamics of propagation of phages, and lead to subpopulations specialized

526 for different spatial niches. A third limitation of the model lies on the lack of a true physiological 
527 description of the bacteria. We assume that phages can infect bacteria at any time, but phage

528 infectivity is known, in some cases, to depend on whether its bacterial host is in exponential or

529 stationary phase [49]. In other cases, a stochastic or induced persistence state in bacteria allows

530 the population to maintain alive a sensitive subpopulation [50]. This can lead to a slowdown or

531 complete halt of infection, particularly in structured environments. Nevertheless, it is important to

532 underline that the model was designed to be easily extensible and further assimilate new

533 mechanisms. Some that are already implemented but not thoroughly explored here include phage

534 resistance based on adaptive immunity (e.g., CRISPR-Cas [32,51]) or mutations affecting the

535 phage host range [52].

536 One of the major conclusions of this work is that spatial structure affects the dynamics of bacterial

537 populations in the face of antibiotic exposure, phage predation or a combination of both. Whilst

538 combining phages and antibiotics is one of the proposed strategies for the clinical use of phage

$539[53,54]$, we show here that the emergence of bacteria resistant to both stressors can be enhanced

540 by structured environments, particularly when antibiotics are not homogeneously distributed, as

541 seems common in natural settings $[55,56]$. This should be taken into account in phage therapy

542 studies.

543 Adaptation of bacterial cells can also be driven by temperate phage. We showed how

544 autotransduction promotes the spread of antibiotic resistance and is affected by different

545 mechanisms. Importantly, we predicted that different community outcomes (coexistence and

546 extinction) can occur by modulating the efficiency of phages' infection, lysogeny and transduction,

547 as well as the structure of the environment. We experimentally confirmed the emergence of co-

548 existence between strains of $S$. aureus in well mixed environments, underlining the power of our

549 approach in generating valid and testable hypotheses. It will be important to further test its

550 predictions, as well to simulate other ecologically (and clinically) relevant scenarios. In particular,

551 it will be crucial to explore how the co-existence between virulent phages and prophages 
552 influences the outcomes of a combined treatment with phage and antibiotics under a range of

553 ecological interactions between the two types of phages. Exploring these and other ecological

554 settings is also key to understand which factors impact the evolutionary consequences of phage-

555 bacteria interactions for microbial populations.

We thank Marie Touchon and José Pénadés for comments, suggestions, and criticisms during the

559 development and testing of the model. This work was funded by European Research Council grant

560 [EVOMOBILOME ${ }^{\circ}$ 281605] to E.P.C.R. and by an EU FP7 PRESTIGE grant [PRESTIGE-2017-

561 1-0012] from Campus France to J.A.M.S. H.I. is supported by the Danish National Research

562 Foundation's Centre of Excellence Bacterial Stress Response and Persistence (DNRF12) and

563 J.H. is supported by a Young Elite Researcher grant from the Danish Research Council, Sapere

564 Aude.

565

\section{REFERENCES}

567 1. Didelot X, Walker AS, Peto TE, Crook DW, Wilson DJ. Within-host evolution of bacterial 568 pathogens. Nat Rev Micro. 2016;14: 150-162. doi:10.1038/nrmicro.2015.13

$5692 . \quad$ Brooks AW, Kohl KD, Brucker RM, van Opstal EJ, Bordenstein SR. Phylosymbiosis:

$570 \quad$ Relationships and Functional Effects of Microbial Communities across Host Evolutionary

$571 \quad$ History. PLoS Biol. 2016;14: e2000225-29. doi:10.1371/journal.pbio.2000225

572 3. Fuhrman JA. Marine viruses and their biogeochemical and ecological effects. Nature.

573 1999;399: 541-548. doi:10.1038/21119

574 4. Koskella B, Brockhurst MA. Bacteria-phage coevolution as a driver of ecological and 575 evolutionary processes in microbial communities. FEMS Microbiol Rev. 2014;38: 916576 931. doi:10.1111/1574-6976.12072

577 5. Perfeito L, Fernandes L, Mota C, Gordo I. Adaptive mutations in bacteria: high rate and $578 \quad$ small effects. Science. 2007;317: 813-815. doi:10.1126/science.1142284 
579 6. Ghoul M, Mitri S. The Ecology and Evolution of Microbial Competition. Trends Microbiol. 2016;24: 833-845. doi:10.1016/j.tim.2016.06.011

581 7. Engelhardt T, Kallmeyer J, Cypionka H, Engelen B. High virus-to-cell ratios indicate ongoing production of viruses in deep subsurface sediments. ISME J. 2014;8: 15031509. doi:10.1038/ismej.2013.245

8. Díaz-Muñoz SL, Koskella B. Bacteria-phage interactions in natural environments. Adv

9. Clokie MR, Millard AD, Letarov AV, Heaphy S. Phages in nature. Bacteriophage. 2011;1:

10. Suttle CA. Marine viruses--major players in the global ecosystem. Nat Rev Micro. 2007;5: 801-812. doi:10.1038/nrmicro1750

11. Roach DR, Debarbieux L. Phage therapy: awakening a sleeping giant. Emerg Top Life

12. Susskind MM, Botstein D, Wright A. Superinfection exclusion by P22 prophage in lysogens of Salmonella typhimurium. III. Failure of superinfecting phage DNA to enter sieA+ lysogens. Virology. 1974;62: 350-366.

13. Harrison E, Brockhurst MA. Ecological and Evolutionary Benefits of Temperate Phage: What Does or Doesn't Kill You Makes You Stronger. Bioessays. 2017;197: 1700112. doi:10.1002/bies.201700112

14. Bondy-Denomy J, Qian J, Westra ER, Buckling A, Guttman DS, Davidson AR, et al. Prophages mediate defense against phage infection through diverse mechanisms. ISME J. 2016;10: 2854-2866. doi:10.1038/ismej.2016.79

15. Touchon M, Bernheim A, Rocha EP. Genetic and life-history traits associated with the

16. Brüssow H, Canchaya C, Hardt W-D. Phages and the evolution of bacterial pathogens:

17. Bossi L, Fuentes JA, Mora G, Figueroa-Bossi N. Prophage contribution to bacterial from genomic rearrangements to lysogenic conversion. Microbiol Mol Biol Rev. 2004;68: 560-602. doi:10.1128/MMBR.68.3.560-602.2004

615 20. Weinstock GM. Transduction in Gram-Negative Bacteria. Modern Microbial Genetics. 
617 21. Henry M, Debarbieux L. Tools from viruses: bacteriophage successes and beyond. Virology. 2012;434: 151-161. doi:10.1016/j.virol.2012.09.017

22. Kenzaka T, Tani K, Sakotani A, Yamaguchi N, Nasu M. High-frequency phage-mediated gene transfer among Escherichia coli cells, determined at the single-cell level. Appl Environ Microbiol. 2007;73: 3291-3299. doi:10.1128/AEM.02890-06

23. Kenzaka T, Tani K, Nasu M. High-frequency phage-mediated gene transfer in freshwater environments determined at single-cell level. ISME J. 2010;4: 648-659. doi:10.1038/ismej.2009.145

24. Xia G, Wolz C. Phages of Staphylococcus aureus and their impact on host evolution.

25. Balcazar JL. Bacteriophages as Vehicles for Antibiotic Resistance Genes in the Infect Genet Evol. 2014;21: 593-601. doi:10.1016/j.meegid.2013.04.022

26. Haaber J, Penadés JR, Ingmer H. Transfer of Antibiotic Resistance in Staphylococcus

27. Haaber J, Leisner JORJ, Cohn MT, Catalan-Moreno A, Nielsen JB, Westh H, et al.

28. Bull JJ, Gill JJ. The habits of highly effective phages: population dynamics as a Bacterial viruses enable their host to acquire antibiotic resistance genes from neighbouring cells. Nat Commun. 2016;7: 1-8. doi:10.1038/ncomms13333

29. De Paepe M, Tournier L, Moncaut E, Son O, Langella P, Petit M-A. Carriage of $\lambda$ Latent

30. Weitz JS, Hartman H, Levin SA. Coevolutionary arms races between bacteria and Intestine. PLoS Genet. 2016;12: e1005861. doi:10.1371/journal.pgen.1005861

652 34. McVay CS, Velásquez M, Fralick JA. Phage therapy of Pseudomonas aeruginosa 
653

654

655

656

657

658

659

660

661

662

663

664

665

666

667

668

669

670

671

672

673

674

675

676

677

678

679

680

681

682

683

684

685

686

687

688

689

690

infection in a mouse burn wound model. Antimicrob Agents Chemother. 2007;51: 19341938. doi:10.1128/AAC.01028-06

35. Brockhurst MA, Buckling A, Rainey PB. Spatial heterogeneity and the stability of hostparasite coexistence. J Evol Biol. 2006;19: 374-379. doi:10.1111/j.14209101.2005.01026.x

36. Simmons M, Drescher K, Nadell CD, Bucci V. Phage mobility is a core determinant of phage-bacteria coexistence in biofilms. ISME J. 2017;89: 135.

doi:10.1038/ismej.2017.190

37. Roach DR, Leung CY, Henry M, Morello E, Singh D, Di Santo JP, et al. Synergy between the Host Immune System and Bacteriophage Is Essential for Successful Phage Therapy against an Acute Respiratory Pathogen. Cell Host Microbe. 2017;22: 38-47.e4. doi:10.1016/j.chom.2017.06.018

38. Barr JJ. A bacteriophages journey through the human body. Immunol Rev. 2017;279: 106-122. doi:10.1111/imr.12565

39. Volkova VV, Lu Z, Besser T, Gröhn YT. Modeling the infection dynamics of bacteriophages in enteric Escherichia coli: estimating the contribution of transduction to antimicrobial gene spread. Appl Environ Microbiol. 2014;80: 4350-4362.

doi:10.1128/AEM.00446-14

40. Hellweger FL, Clegg RJ, Clark JR, Plugge CM, Kreft J-U. Advancing microbial sciences by individual-based modelling. Nat Rev Micro. 2016;14: 461-471. doi:10.1038/nrmicro.2016.62

41. Nadell CD, Foster KR, Xavier JB. Emergence of Spatial Structure in Cell Groups and the Evolution of Cooperation. PLoS Comput Biol. 2010;6: e1000716-9. doi:10.1371/journal.pcbi.1000716

42. Grimm V, Berger U, Bastiansen F, Eliassen S, Ginot V, Giske J, et al. A standard protocol for describing individual-based and agent-based models. Ecol Modell. 2006;198: 115126. doi:10.1016/j.ecolmodel.2006.04.023

43. Andersson DI, Levin BR. The biological cost of antibiotic resistance. Curr Opin Microbiol. 1999;2: 489-493.

44. Samson JE, Magadán AH, Sabri M, Moineau S. Revenge of the phages: defeating bacterial defences. Nat Rev Micro. 2013;11: 1-13. doi:10.1038/nrmicro3096

45. Gómez P, Ashby B, Buckling A. Population mixing promotes arms race host-parasite coevolution. Proc Biol Sci. 2015;282: 20142297-20142297. doi:10.1098/rspb.2014.2297

46. Zeng L, Skinner SO, Zong C, Sippy J, Feiss M, Golding I. Decision making at a subcellular level determines the outcome of bacteriophage infection. Cell. 2010;141: 682691. doi:10.1016/j.cell.2010.03.034

47. Brown SP, Le Chat L, De Paepe M, Taddei F. Ecology of microbial invasions:

amplification allows virus carriers to invade more rapidly when rare. Curr Biol. 2006;16: 
692 48. Lang AS, Westbye AB, Beatty JT. The Distribution, Evolution, and Roles of Gene Transfer Agents in Prokaryotic Genetic Exchange. Annu Rev Virol. 2017;4: 87-104. doi:10.1146/annurev-virology-101416-041624

49. Chibani-Chennoufi S, Bruttin A, Dillmann M-L, Brüssow H. Phage-host interaction: an ecological perspective. J Bacteriol. American Society for Microbiology; 2004;186: 36773686. doi:10.1128/JB.186.12.3677-3686.2004

50. Chapman-McQuiston E, Wu XL. Stochastic receptor expression allows sensitive bacteria to evade phage attack. Part I: experiments. Biophys J. 2008;94: 4525-4536. doi:10.1529/biophysj.107.120212

51. Bradde S, Vucelja M, Teşileanu T, Balasubramanian V. Dynamics of adaptive immunity against phage in bacterial populations. PLoS Comput Biol. 2017;13: e1005486-16. doi:10.1371/journal.pcbi.1005486

52. De Sordi L, Khanna V, Debarbieux L. The Gut Microbiota Facilitates Drifts in the Genetic Diversity and Infectivity of Bacterial Viruses. Cell Host Microbe. 2017;22: 801-808.e3. doi:10.1016/j.chom.2017.10.010

53. Torres-Barceló C, Hochberg ME. Evolutionary Rationale for Phages as Complements of

54. Arias-Sánchez FI, Allen RC, Hall AR. Effects of prior exposure to antibiotics on bacterial adaptation to phages. J Evol Biol. 2018;11: 2970-10. doi:10.1111/jeb.13220

55. Anderl JN, Franklin MJ, Stewart PS. Role of antibiotic penetration limitation in Klebsiella pneumoniae biofilm resistance to ampicillin and ciprofloxacin. Antimicrob Agents Chemother. 2000;44: 1818-1824.

56. Pratama AA, van Elsas JD. The "Neglected" Soil Virome - Potential Role and Impact. Trends Microbiol. 2018;: 1-14. doi:10.1016/j.tim.2017.12.004 
A

Spatially
Structured

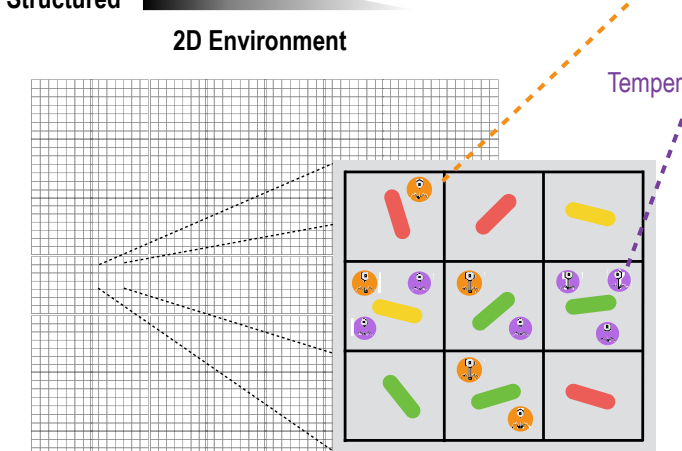

Temperate phage

|

$$
\text { . }
$$

Antibiotic resistan

Antibiotic sensitive

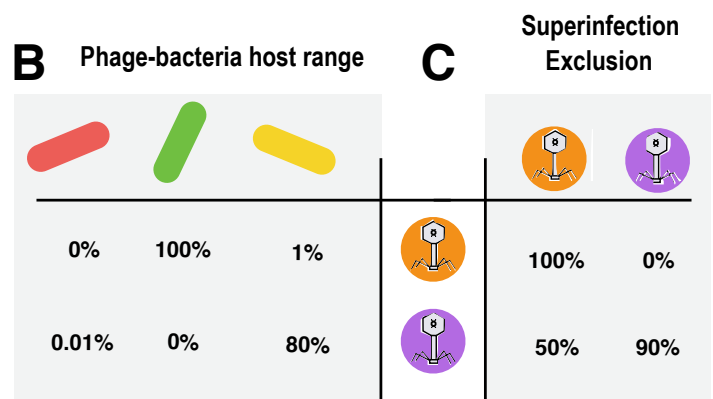

D Death and selection by antibiotics

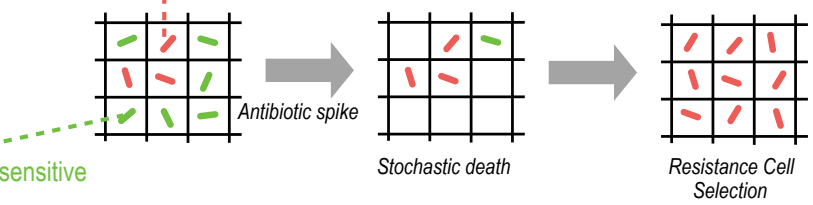

E Bacterial Selection and Replication

$\mathbf{F}$

Infection and Transduction of Bacterial Traits

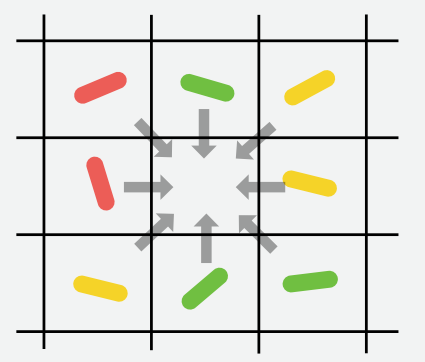

Roulette wheel probability proportional
to reproductive fitness
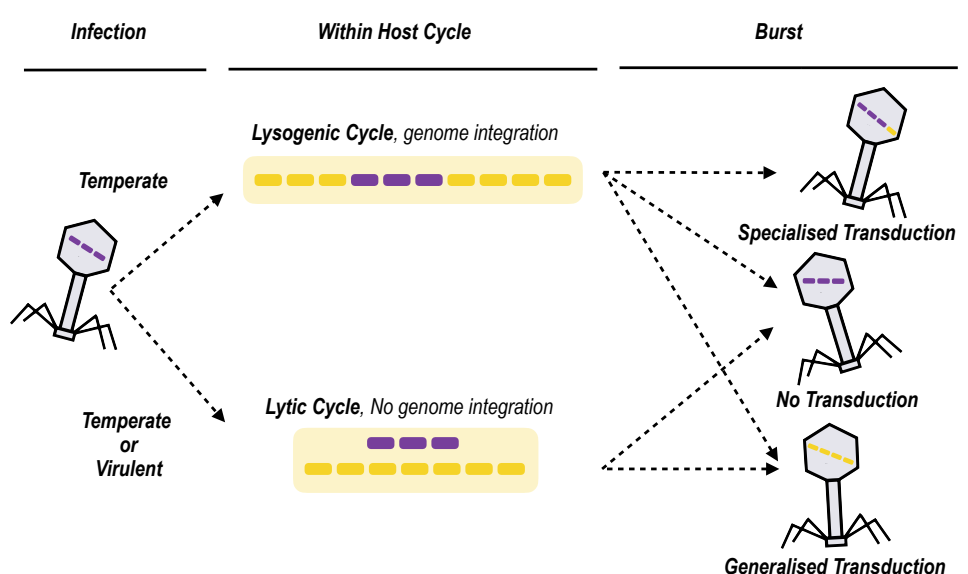

G

을

- Bacteria species/types

- Phage species/types

Phage/Bacteria Infection matrix

Superinfection Protection matrix

- Environmental Size/Structure

- Antibiotic treatment schedule

$\rightarrow \quad$ Collect Population Statistics $\downarrow$

Antibiotic Application (If scheduled)

$$
\downarrow
$$

Randomise Population Access Order

$\downarrow$
Bacterial Death

- Calculate probability of death

- Intrinsic

- Phage induction and lysis

- Release lysed phage into the environment

- Remove cells and free resources (space)

$\frac{1}{0}$
0
$\frac{1}{\pi}$
$\frac{1}{2}$

\section{$\downarrow$ \\ Phage Infection}

Decision between lytic and lysogenic cycle for temperate phage

$$
\downarrow
$$

Selection and Reproduction Probability of reproduction followed by roulette wheel selection

\section{$\downarrow$}

Randomise Cell Locations

(If environment is defined as "Well-Mixed") $\downarrow$

Environment Update

- Phage dispersal and decay

- Antibiotics dispersal and decay 


\section{Well Mixed Environment}

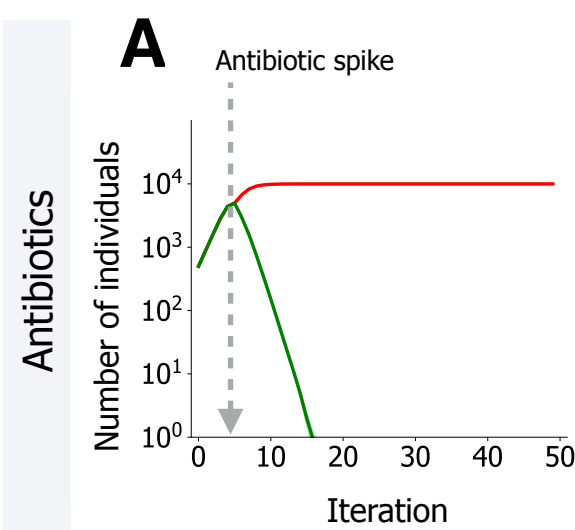

Antibiotic resistant/phage sensitive bacteria

Phage resistant/antibiotic sensitive bacteria

Free phage particles

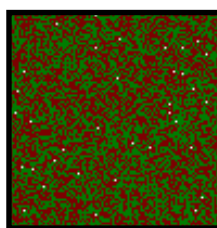

$t=5$

B
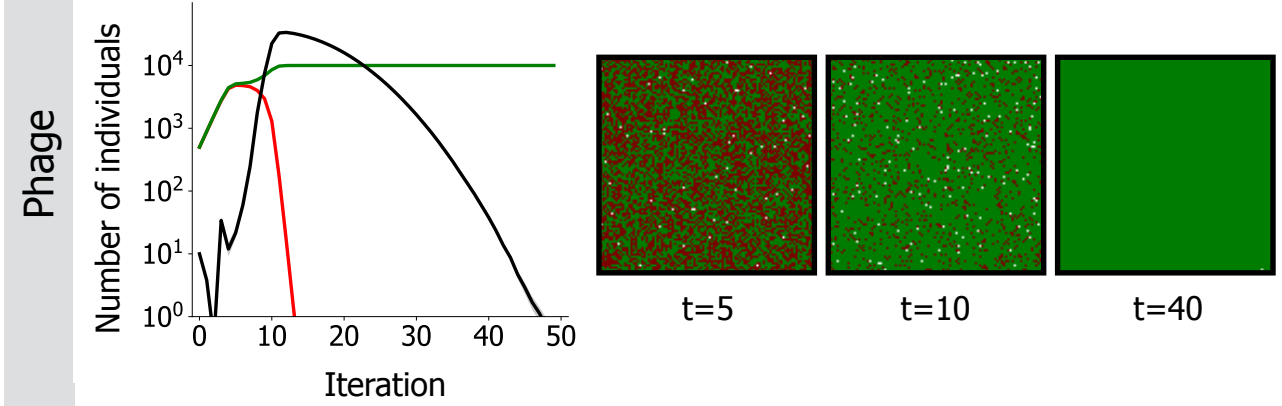

C Antibiotic spike
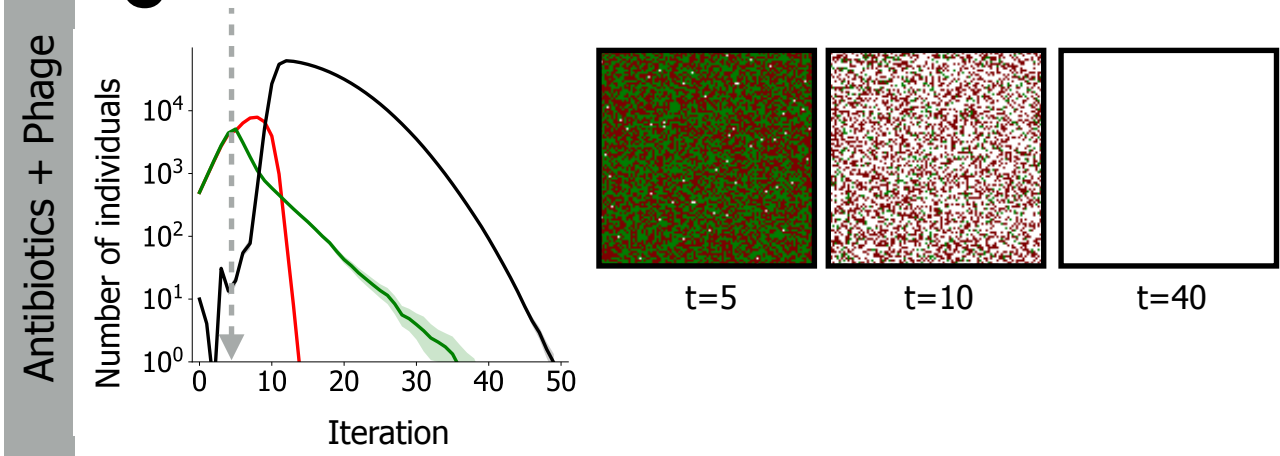

Spatially Structured Environment

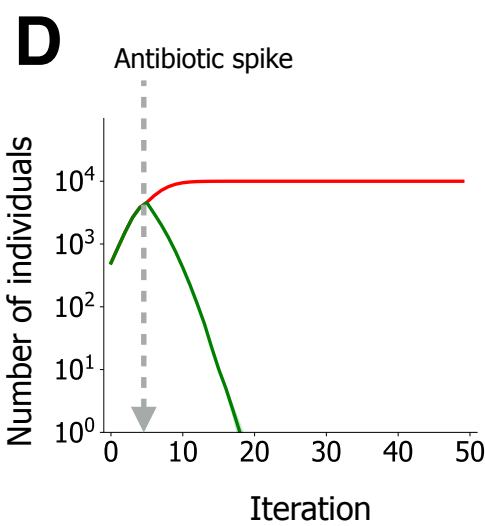

Antibiotic resistant/phage sensitive bacteria Free phage particles

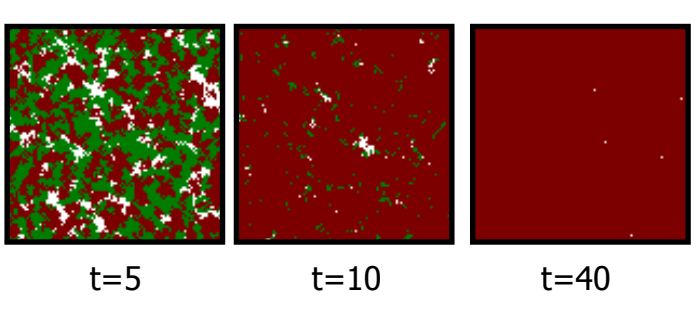

E
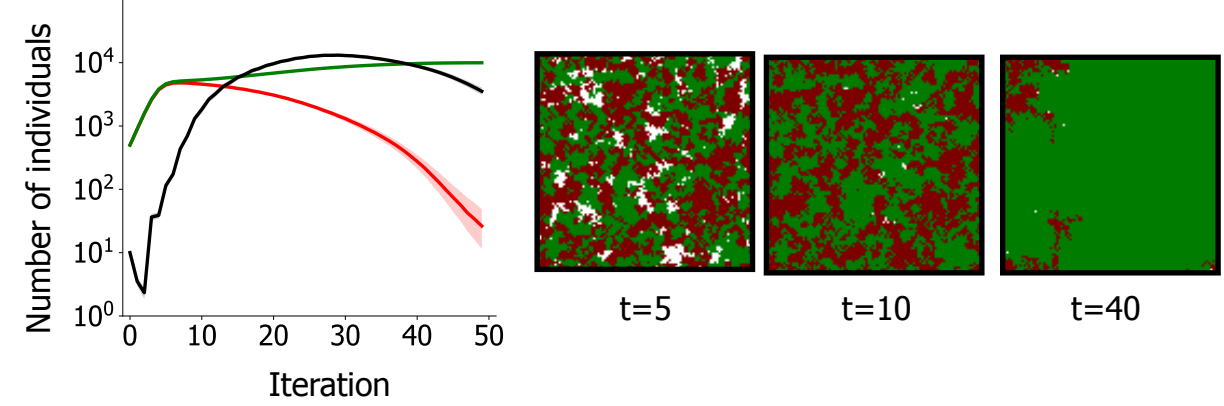

$\mathbf{F}$

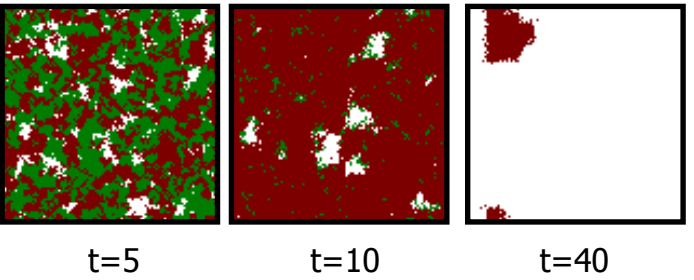

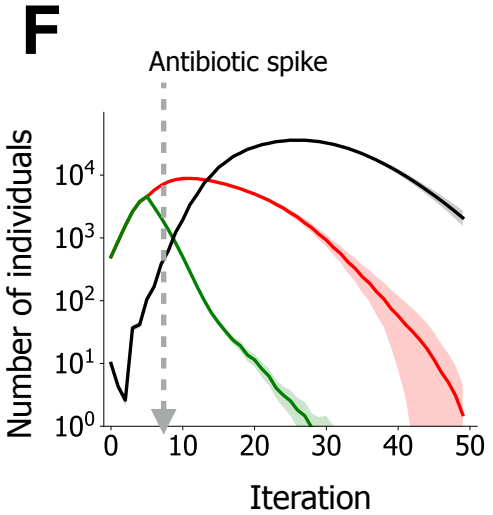




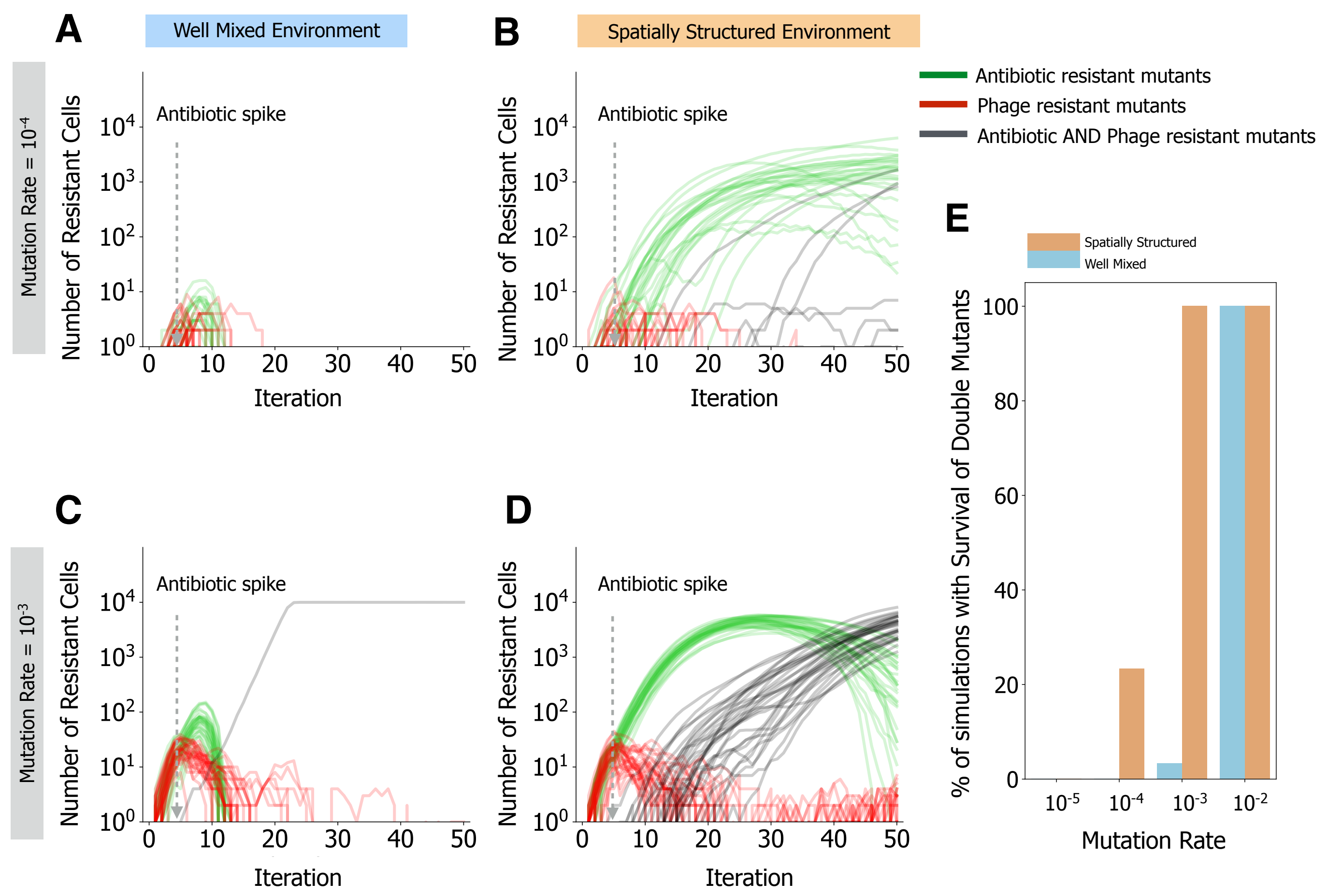



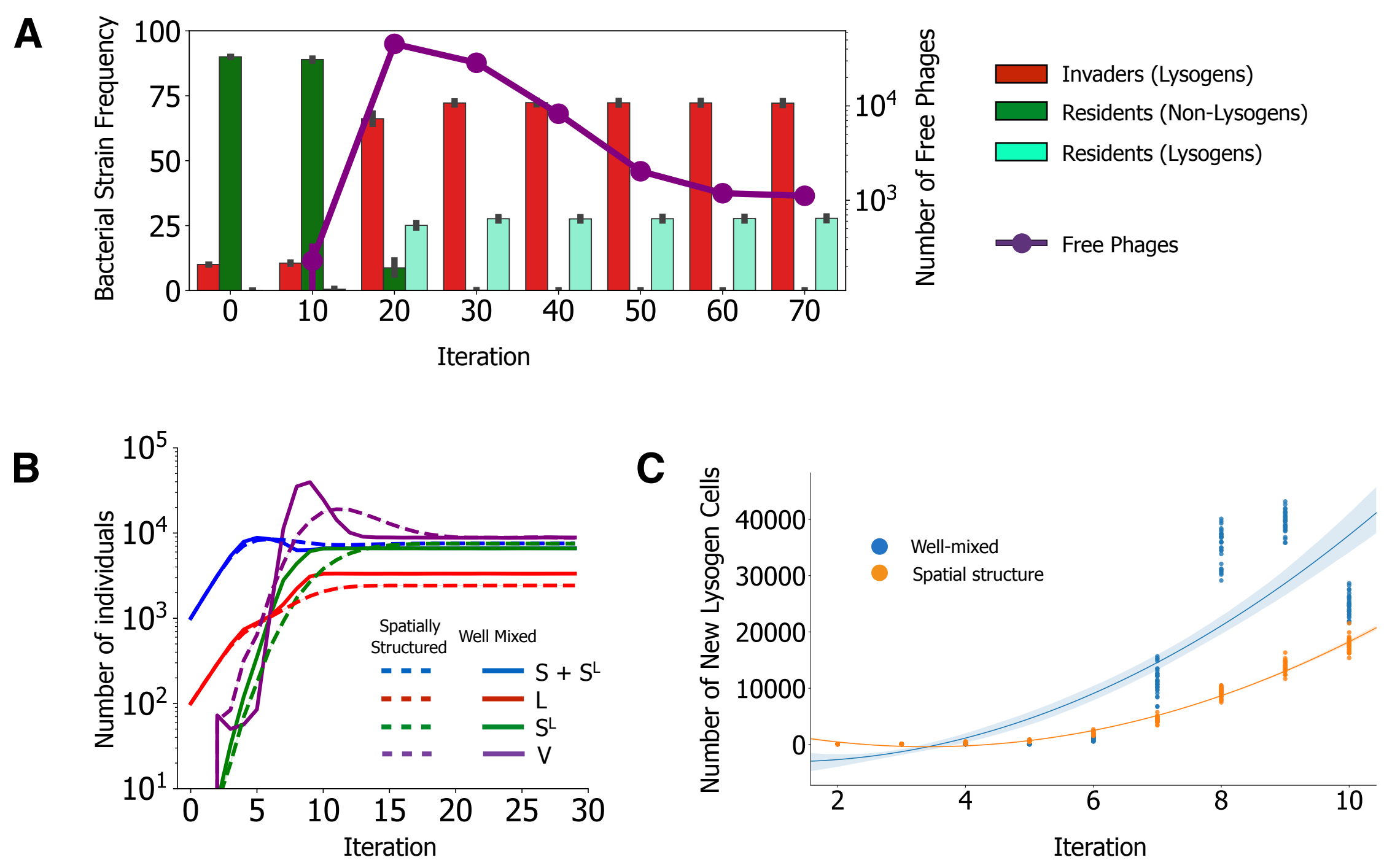
Fig 5

A

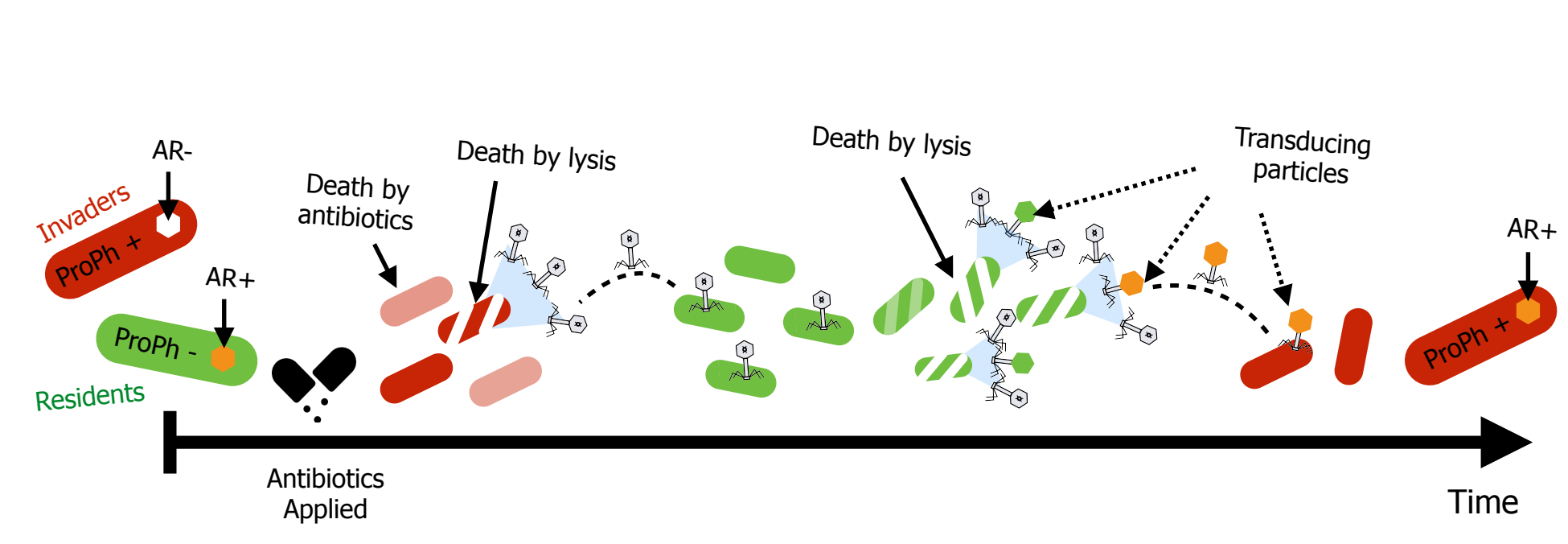

C
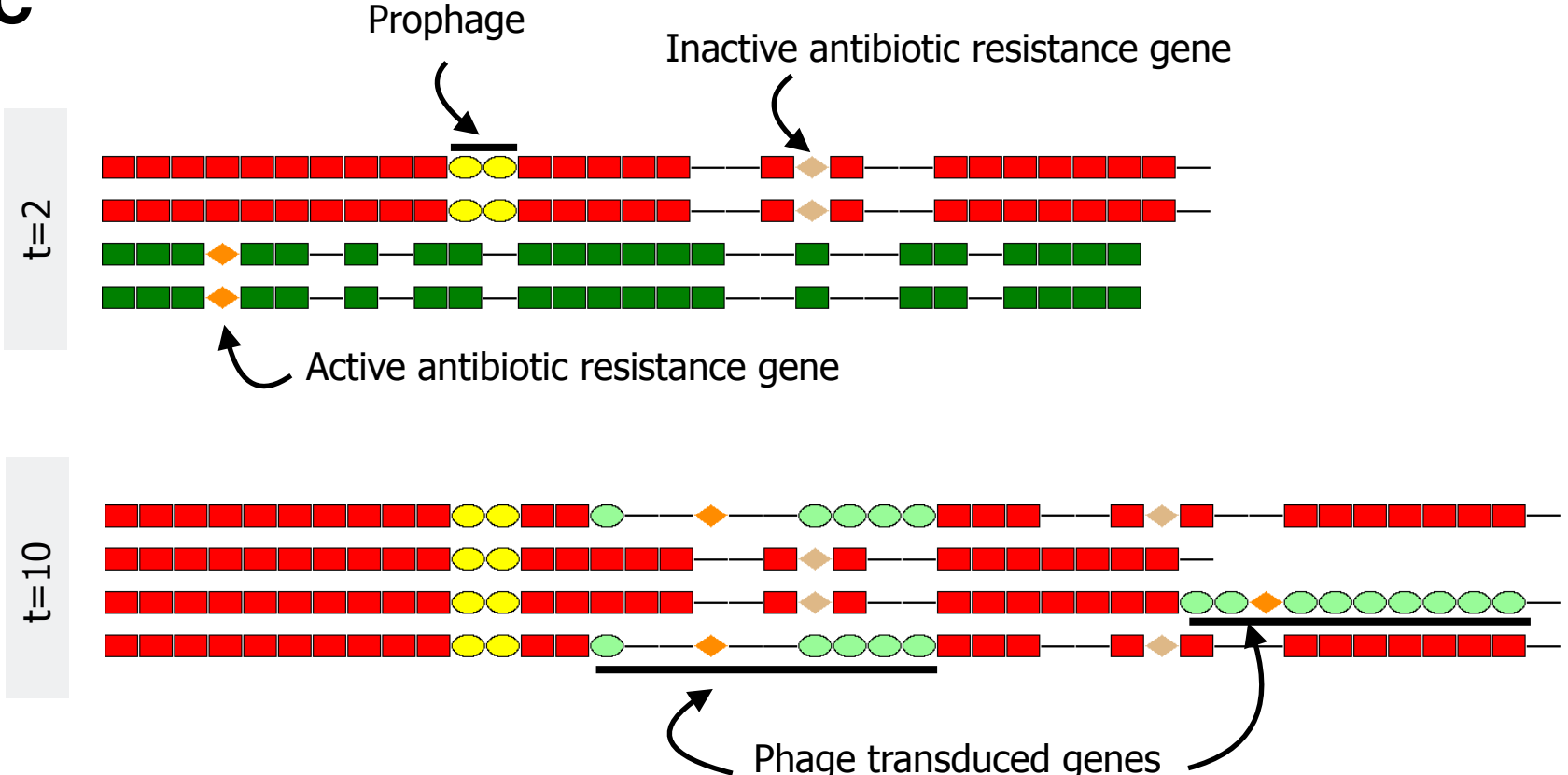

B

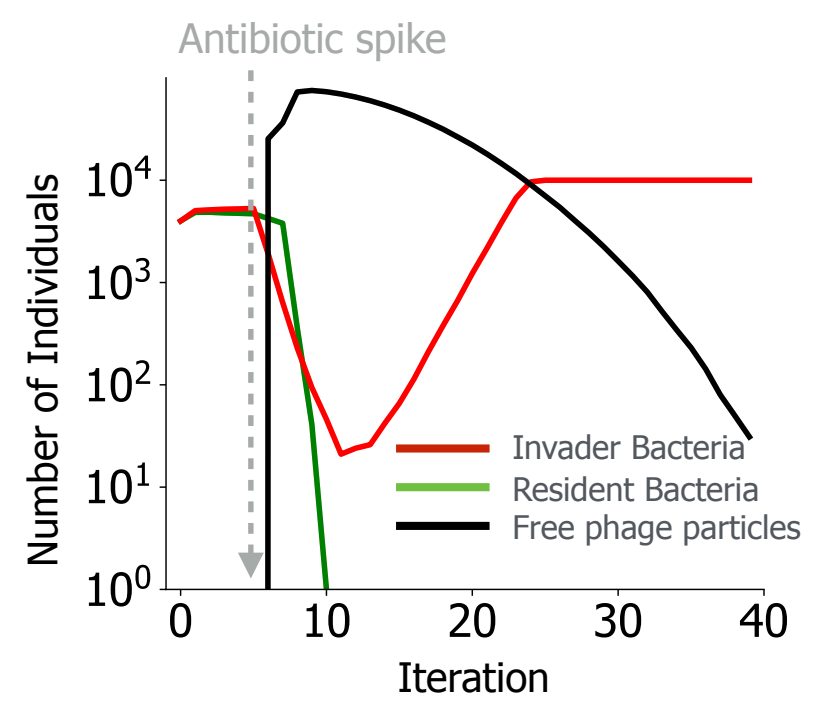

D

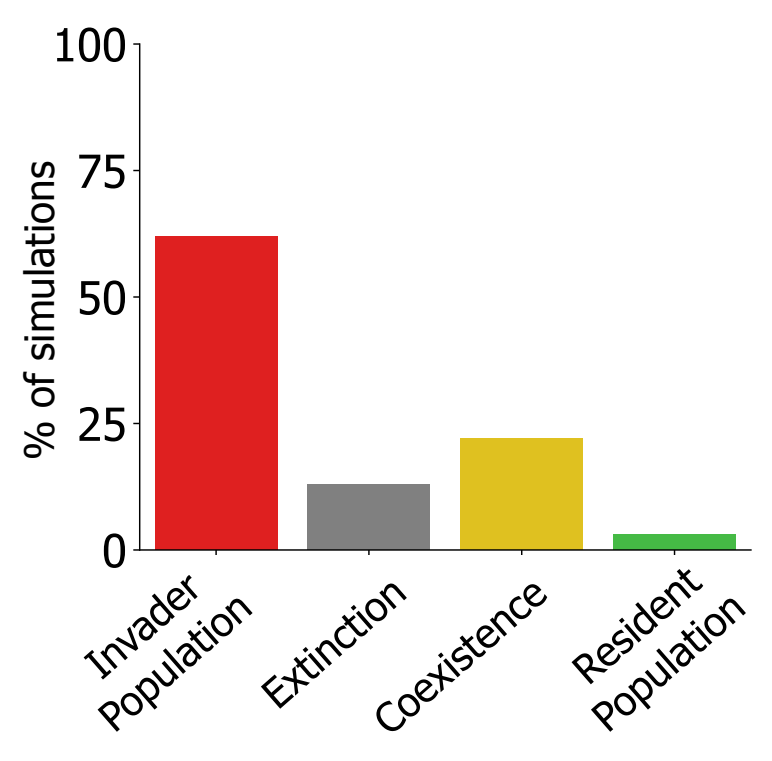


A

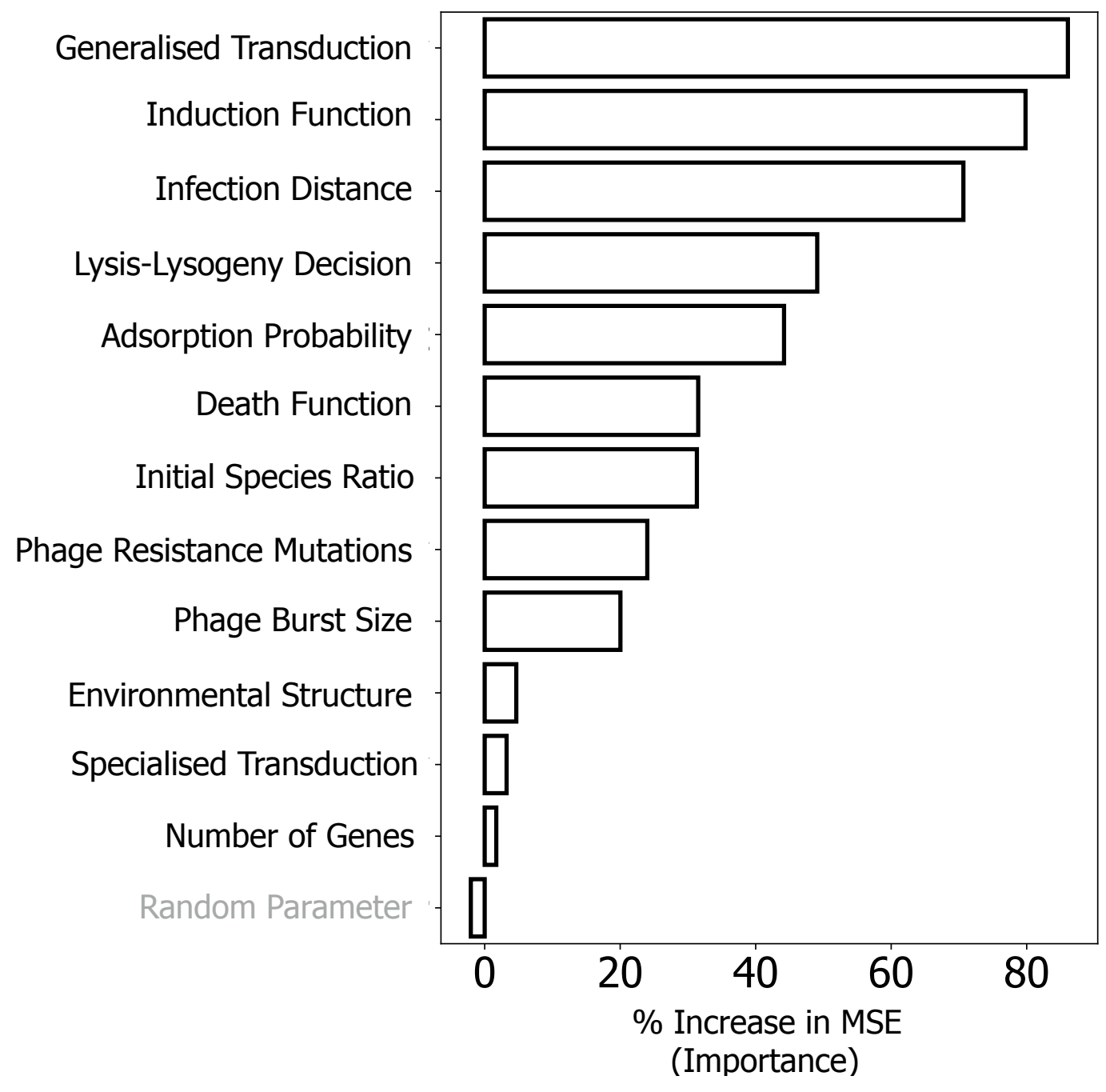

B

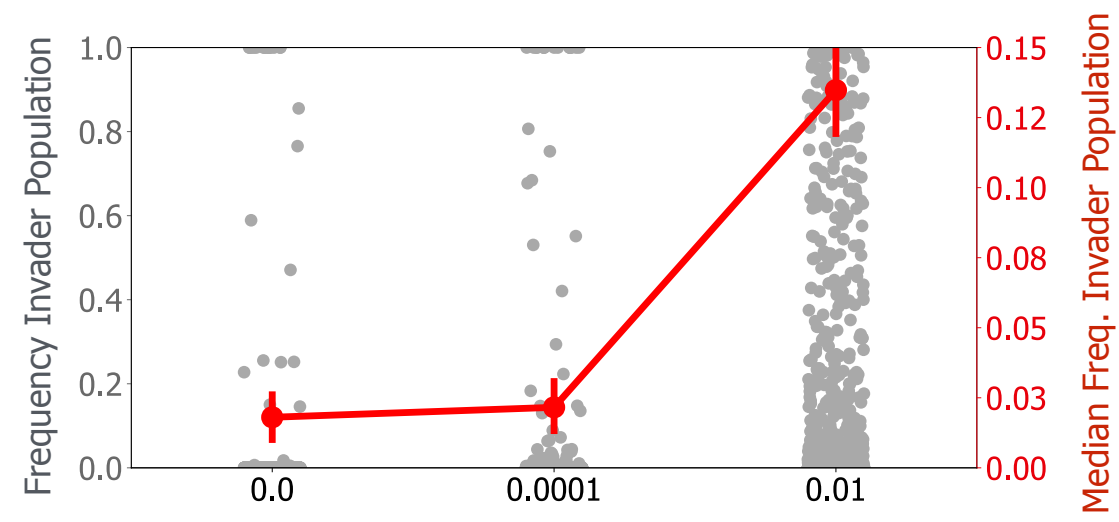

C Probability of Generalised Transduction

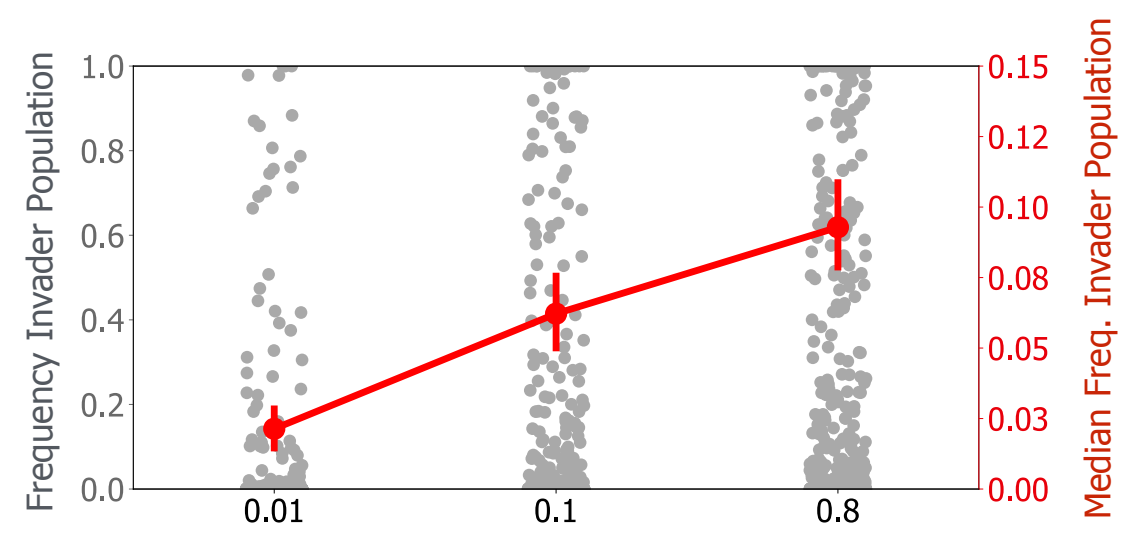

Adsorption Probability 
A

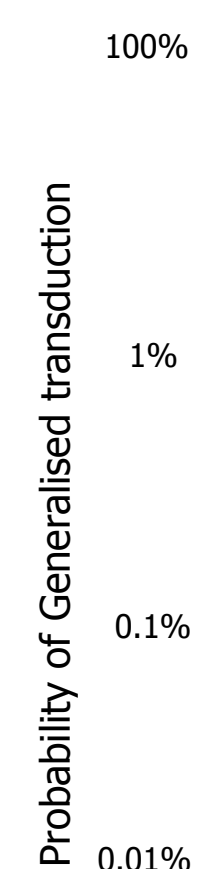

$0.001 \%$
Survival of

Invader

Population

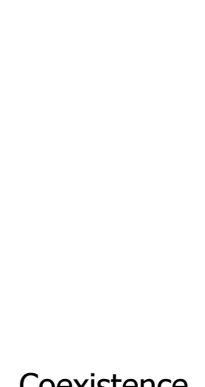

Survival of Resident

Population

Extinction

\section{B}

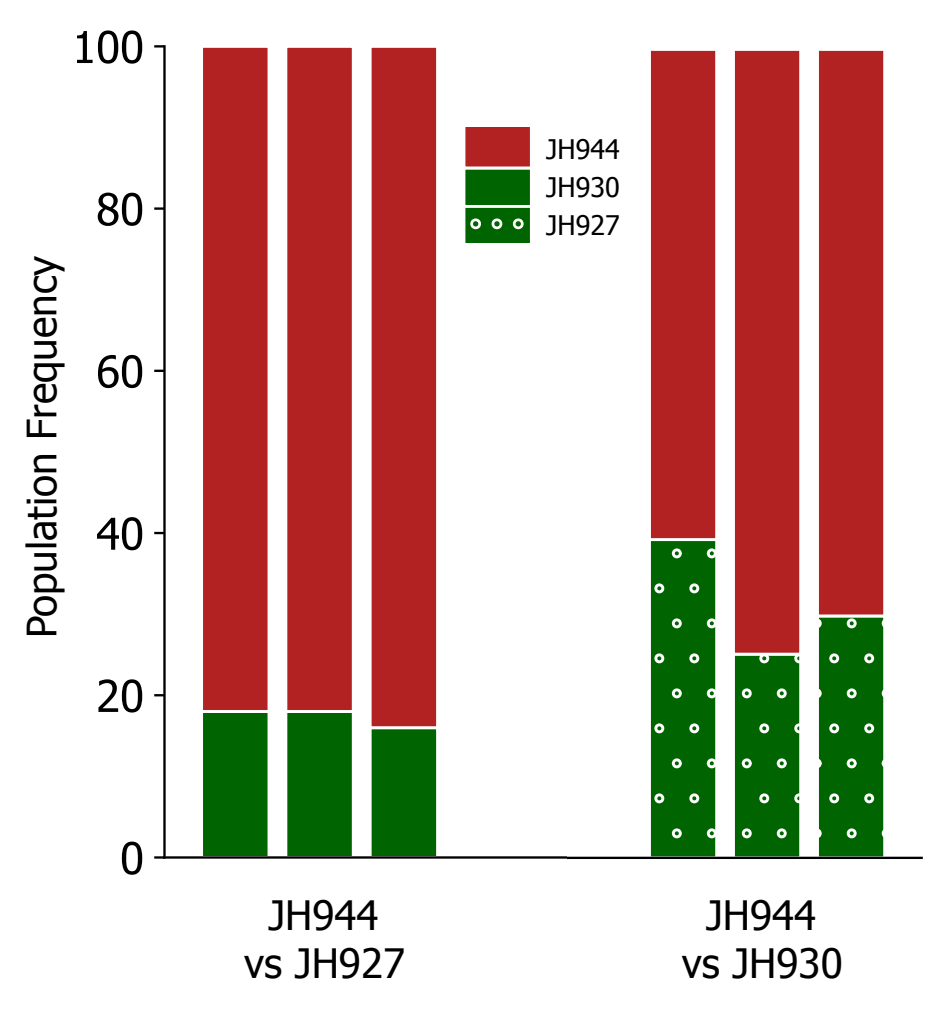

Probability of Adsorption 